\title{
High Power silicon \\ MMIC design for wireless base stations
}

\author{
Gérard Bouisse \\ Motorola Semiconducteurs \\ Avenue du general Eisenhower \\ 31000 Toulouse-France
}

Gerard.Bouisse@motorola.com

\begin{abstract}
This article presents the specificities of high power silicon MMIC, intended for wireless base station applications. Motorola's LDMOS technology, and its integrated version (HVIC), are presented, with emphasis on the particularities of high power functions. The technical problems linked to the design of multistages power amplifiers, of $10 \mathrm{~W}$ and $30 \mathrm{~W}$ output power are listed, and solutions are proposed. Two power amplifier circuits , a 900 $\mathrm{MHz} 3$ stages $30 \mathrm{~W}$ and a $1800 \mathrm{MHz} 3$ stages $10 \mathrm{~W}$ and their associated performances demonstrate the validity of these solutions.
\end{abstract}

\section{I.TECHNOLOGY}

When it comes to power amplification for wireless base station, LDMOS is the technology of choice. Indeed, the supply voltage (26V) and the power levels of interest (a few watts to several hundreds)lead to electrical constraints (such as breakdown voltages) and thermal constraints (very low thermal resistances) that GaAs devices have a hard time to reach. LDMOS is a silicon MOSFET technology with the following properties:

-breakdown voltages in the order of $70 \mathrm{~V}$ -0.6 microns gate length .

-thermal resitances as low as a few tenth of degrees/W.

In consequence LDMOS is a transistor technology perfectly suited for power amplification up to $150 \mathrm{~W}$ at 1 and $2 \mathrm{GHz}$. The association of passive components on Silicium has triggered the design of multistage power amplifiers.
The microstrip transmission lines and the inductances are the limiting components because of their very low quality coefficient, in the order of 5 to 6 , for frequencies between 1 and $2 \mathrm{GHz}$. The coupling by proximity to the low resistivity substrate is responsible for this single digit $\mathrm{Q}$.

\section{II.SPECIFIC PROBLEMS}

Reaching power levels in the order of 10 to $15 \mathrm{~W}$ requires transistors with a gate periphery of $30 \mathrm{~mm}$ to $40 \mathrm{~mm}$, 30W corresponds to an $80 \mathrm{~mm}$ device. These devices feature RF gate and drain impedances around 1 or 2 ohms. Their physical size ranges between 2000 and 5000 microns in the $\mathrm{Y}$-axis and 200 microns in the $\mathrm{X}$-axis. The reactive elements used for impedance matching purposes exhibit much smaller dimensions, around 100 microns. In addition to that, the line width for transmission lines and inductances (and then the connection width) varies between 5 and 30 microns.

This creates several problems:

-The matching loss are prohibitive because of the low $\mathrm{Q}$ of the transmission lines and inductances on one hand, and the very low impedances of the transistors on the other hand.

-The major size difference between actives and passives makes impossible the in phase excitation of the transistor fingers. This is incompatible with power generation at the output of the transistor. 
-Inaccurate simulations result because of the excessive scaling ratio.

\section{III.PROPOSED SOLUTIONS}

Several techniques allow the designer to get around, or at least attenuate the above mentionned problems. Shrinking the gate periphery of the individual transistors, and combining them in a branched amplifier structure represents the key to success. The following advantages result :

-Matching loss major decrease because of two reasons:

*Transistor impedance level increase *matching networks duplication.

-Output power increase because of a better in phase excitation of the different fingers, due to the aspect ratio difference reduction between passives and actives.

-Thermal resistance improvement, or MTTF increase, because of the transistor splits.

There is a possibility to optimize a little bit further by increasing the unit finger width, which reduces further the size difference (in the Y-axis) between actives and passives and then improves the in phase excitation.

\section{IV.PRACTICAL EXAMPLES}

The above presented techniques have allowed 2 circuits design :

-A 3 stages amplifier, in the $1.805 \mathrm{GHz}-$ $1.880 \mathrm{GHz}$ band, featuring $15 \mathrm{~W} 1 \mathrm{~dB}$ compression point. The line-up is based on a $5 \mathrm{~mm}$ fet driving a $10 \mathrm{~mm}$ fet driving two $20 \mathrm{~mm}$ fets.

-A 3 stages amplifier, in the $925 \mathrm{MHz}-960 \mathrm{MHz}$ band, featuring $30 \mathrm{~W}$ compression point and using a $5 \mathrm{~mm}$ fet to drive two $5 \mathrm{~mm}$ fets, driving four $20 \mathrm{~mm}$ fets.

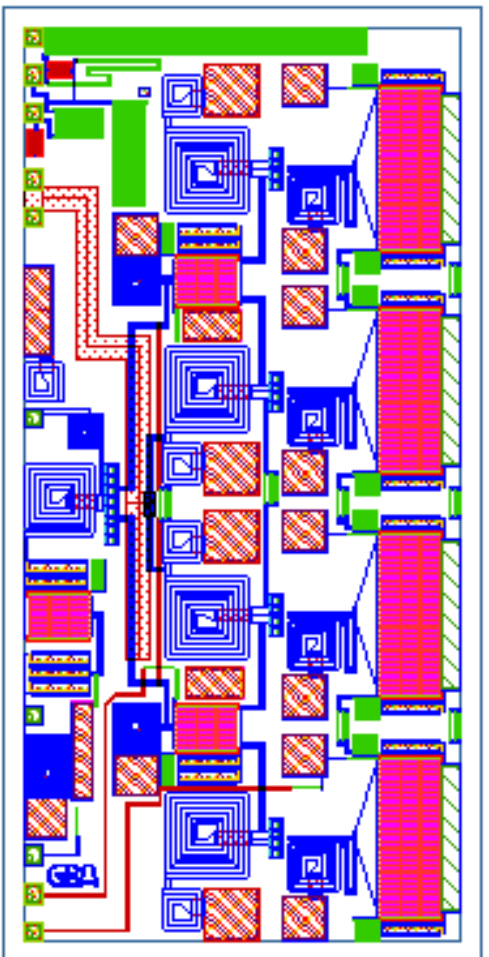

Fig1: 30W 900MHz amplifier layout.

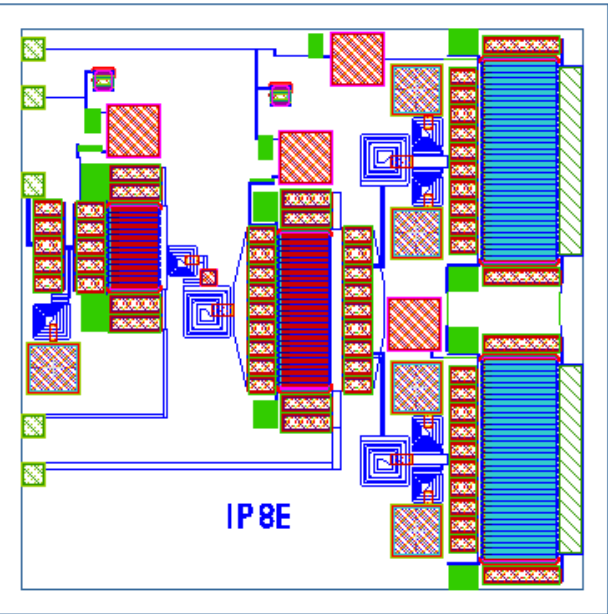

fig 2: $15 \mathrm{~W} 1.8 \mathrm{GHz}$ amplifier layout

These two amplifier performances are presented in figures 3 to 6 . 


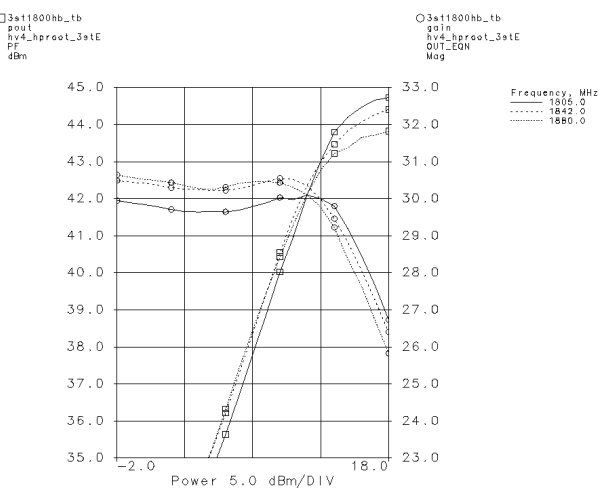

fig3: $1.8 \mathrm{GHz}$ amplifier :gain and power

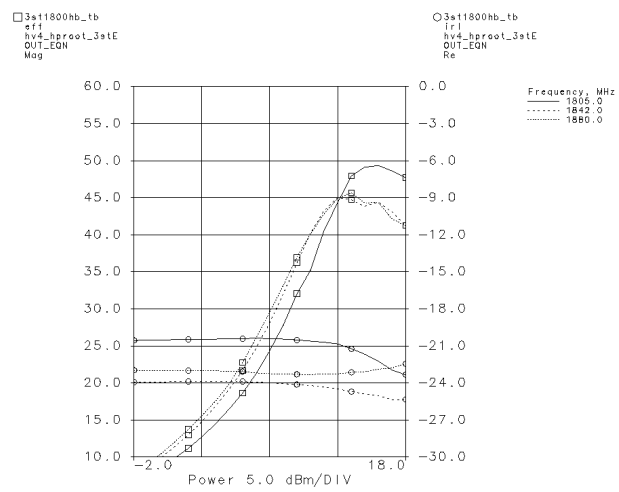

fig4 : $1.8 \mathrm{GHz}$ amplifier : Power Added Efficiency and S11.

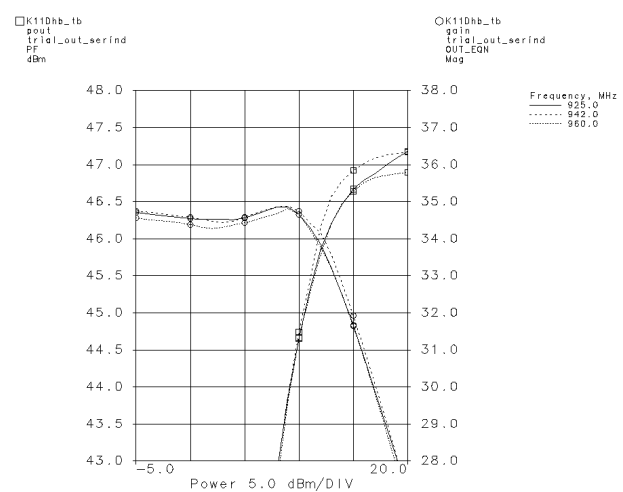

fig 5 : 900MHz amplifier :gain and power

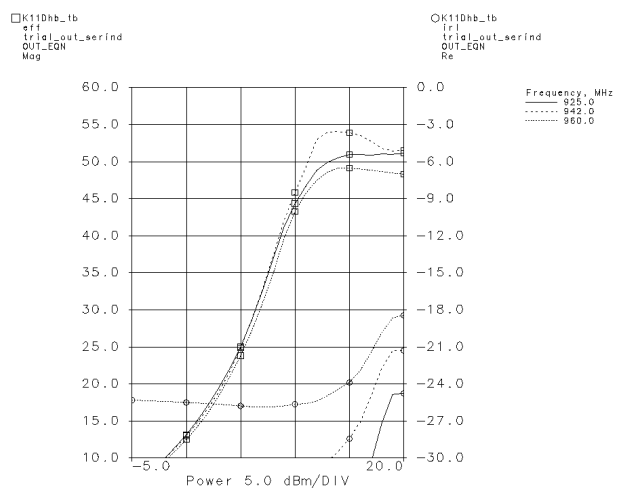

fig $6: 900 \mathrm{MHz}$ amplifier :PAE and S11.

\section{V.CONCLUSION}

The possibility to reach several tens of watts using Silicon MMIC has been demonstrated in this article. Nevertheless, it is necessary to use branched structures amplifiers, using limited gate periphery transistors, along with optimized unit finger width. In addition to the electrical advantages, or simply to the possibility to realize a circuit, these techniques lead to thermal improvements.

It is mandatory for the designer to pay a specific attention to the stability analysis, mainly because of the presence of loops .To that extent the Normalized Determinant Factor technique represents the perfect tool. 